\title{
QUEEN'S
UNIVERSITY
BELFAST
}

\section{A low-cost digital image correlation technique for characterising the shear deformation of fabrics for draping studies}

Pierce, R., Falzon, B. G., Thompson, M. C., \& Boman, R. (2015). A low-cost digital image correlation technique for characterising the shear deformation of fabrics for draping studies. Strain, 51(3), 180-189.

https://doi.org/10.1111/str.12131

\section{Published in:}

Strain

\section{Document Version:}

Peer reviewed version

Queen's University Belfast - Research Portal:

Link to publication record in Queen's University Belfast Research Portal

\section{Publisher rights}

Copyright 2015 Wiley Publishing.

This is the peer reviewed version of the following article: Pierce, RS, Falzon, BG, Thompson, MC, and Boman, R (2015), A Low-Cost Digital Image Correlation Technique for Characterising the Shear Deformation of Fabrics for Draping Studies. Strain, 51, 180-189, which has been published in final form at doi: 10.1111/str.12131. This article may be used for non-commercial purposes in accordance with Wiley Terms and Conditions for Self-Archiving.

\section{General rights}

Copyright for the publications made accessible via the Queen's University Belfast Research Portal is retained by the author(s) and / or other copyright owners and it is a condition of accessing these publications that users recognise and abide by the legal requirements associated with these rights.

Take down policy

The Research Portal is Queen's institutional repository that provides access to Queen's research output. Every effort has been made to ensure that content in the Research Portal does not infringe any person's rights, or applicable UK laws. If you discover content in the Research Portal that you believe breaches copyright or violates any law, please contact openaccess@qub.ac.uk. 


\section{A low-cost digital image correlation technique for characterising the shear deformation of fabrics for draping studies}

Robert S. Pierce ${ }^{a}$, Brian G. Falzon ${ }^{a, b^{*}}$, Mark C. Thompson ${ }^{\mathrm{a}}$ and Romain Boman ${ }^{\mathrm{c}}$

a Department of Mechanical and Aerospace Engineering, Monash University, Melbourne, VIC 3800, Australia

${ }^{\mathrm{b}}$ School of Mechanical and Aerospace Engineering, Queen's University Belfast, United Kingdom, BT9 5AH

${ }^{c}$ Aerospace and Mechanical Engineering Department, University of Liege, Belgium

* Corresponding author. Address: School of Mechanical and Aerospace Engineering, Queen's University

Belfast, United Kingdom, BT9 5AH. Tel.: +44 (0)28 9097 5640. Email: b.falzon@qub.ac.uk 


\begin{abstract}
A novel Digital Image Correlation (DIC) technique has been developed to track changes in textile yarn orientations during shear characterisation experiments, requiring only low-cost digital imaging equipment. Fabric shear angles and effective yarn strains are calculated and visualised using this new DIC technique for bias extension testing of an aerospace grade, carbon-fibre reinforcement material with a plain weave architecture. The DIC results are validated by direct measurement, and the use of a wide bias extension sample is evaluated against a more commonly-used narrow sample. Wide samples exhibit a shear angle range $25 \%$ greater than narrow samples and peak loads which are ten times higher. This is primarily due to excessive yarn slippage in the narrow samples, hence the wide sample configuration is recommended for characterisation of shear properties which are required for accurate modelling of textile draping.
\end{abstract}

Keywords: Digital image correlation, Fabrics/textiles, Mechanical testing, Shear behaviour.

\title{
Introduction
}

In recent years, the characterisation of fabric shear properties has become increasingly important as a result of growing interest in textile-reinforced composite materials. Principally, these materials show greater damage tolerance, improved out-of-plane mechanical properties and superior forming capabilities over complex geometries, compared with traditional stacked laminates of uni-directional plies [1]. However, because manufacturing continues to rely on costly and wasteful empirical practices, simulation of fabric deformation behaviour is becoming more common $[2,3]$. The accuracy of these simulations is highly dependent on the quality of the material characterisation.

During textile forming, or draping, the initial shear resistance to deformation is minimal and yarn tension increases, however as yarns reorient themselves, shear resistance grows until an eventual locking state is reached. This produces an exponential increase in shear stiffness, making in-plane shear is the dominant deformation mode for fabric materials $[4,5]$. Once yarns are locked, any further deformation is typically reliant on yarn slippage or out-of-plane buckling, depending on the tensile state of the fabric yarns [2]. These three phases are often distinguishable during shear characterisation of a woven fabric material [5].

Though predictive simulations for fabric properties have been demonstrated [6], non-standardised experimental shear characterisation methods remain prominent. Historically, the Kawabata simple shear test has been used [7], loading one edge of samples both tangentially and perpendicularly, while the opposite edge is clamped. Since the late 1990s though, the picture frame test has become more popular, where square or cruciform samples are clamped into a deformable rhombus-shaped rig, and sheared by loading opposite corners of the frame [8]. Additionally, the 'bias extension' test is used by many researchers where rectangular samples are cut and oriented such that the warp and weft yarns are $\pm 45^{\circ}$ relative to an axial loading direction. In this test, a central diamond shaped region of 'pure' shear is induced. More recently, a biaxial bias extension test was introduced to highlight the significance of transverse bias loading, though this adds further complexity to an already challenging test [2]. Despite the long history of research and ongoing work by an international collaborative effort to establish testing benchmarks [9], there are no test standards in this area.

In the shear testing of textile reinforcement materials, direct measurement techniques like strain gauges are generally inappropriate as they tend to interfere with the behaviour of the material. Similarly, theoretical calculations can be unreliable as they often make generalised and inaccurate assumptions about deformation behaviour, particularly for bias extension testing where sample deformation is not uniform. Hence, 
unobtrusive and reliable optical measurement techniques such as Digital Image Correlation (DIC) are recommended [10].

DIC works by correlating sequential images obtained during testing with a static camera system. Visually discernible features on the specimen surface are tracked through the image sequence, measuring displacement and calculating local strains that result from sample deformation [10]. This typically means that samples require paint speckling in order for the DIC to be able to track distinct features on a visually plain sample, however due to the complex architecture of textile reinforcements there are enough surface features to make this step unnecessary. DIC can achieve a high degree of accuracy for a large range of deformation and can be performed either real-time or as a post-processing operation.

Commercially bundled hardware and software packages are typically used for DIC, which tend to be quite sophisticated and applicable to a large variety of applications, both in 2D and 3D measurements. These packages usually include one or two CCD (Charge-Coupled Device) cameras, connected to a computer running propriety software that performs the actual DIC. Hence, these systems are often expensive. Some researchers have developed software to perform DIC based on images taken from various camera systems [9]. One group has even successfully performed DIC using a flatbed scanner instead of a camera for textile reinforcement material testing [11]. Ultimately, DIC is proving to be the most reliable and accurate way to measure deformation in textile reinforcements during material characterisation testing.

This paper focuses on the development of a novel DIC code specifically for textile material characterisation testing, including a study of wide and narrow sample configurations for the bias extension shear test.

\section{DIC for textile shear characterisation}

Currently, most DIC systems calculate the deformation gradient across samples in order to determine the major and minor strains. In the case of textile shear testing, this means that the user is left to later calculate the fabric shear angle from the deformation gradient, which is not even available from some DIC software [12]. Subsequently, a new code has been developed in MATLAB, specifically for the characterisation of fabric shear properties, based on an open source code for axial strain developed by Eberl [13]. This new DIC code is available on the MathWorks file exchange website with supporting documentation [14].

The new DIC code is designed to determine the in-plane shear response of a textile material, specifically from bias extension testing (though the approach is also applicable to the picture frame experiment). The twodimensional test method requires only a single camera to capture the necessary sequential images for DIC. A three-dimensional, dual camera system is less appropriate as there can be issues with the reflections and shadows that result from textile waviness [12]. However, this does mean that out-of-plane material deformation and buckling cannot be measured with a single camera system. Elaborate calibration is also unnecessary since the shear deformation is simply a relative angular change in yarn directions (though a scale calibration can be used to determine effective yarn strains). The only requirement of the DIC code is that the camera remains static and perpendicular to samples during testing, with sufficient resolution to distinguish features for tracking.

\section{Tracking displacement}

The DIC code works by first collating a series of sequential images and extracting the time information from the meta-data of each image. The gauge region of interest is selected and discretised into an initial correlation 
point grid. Pairs of images (an initial 'base' image and a consecutive 'input' image) are iteratively taken from the image series and correlated, along with the reference grid for the base image, in order to find a displaced grid of points for the input image. Within this iterative 'image loop', the two images are each broken down into smaller elements to enhance the efficiency of the cross-correlation. Then within a nested 'grid point loop', for each correlation point in the grid, the associated elements from the two images are analysed using normalised 2D cross-correlation according to the work by Lewis [15] (correlating the two sub-images using either the 2D convolution in the spatial domain or 2D discrete Fourier transforms in the frequency domain, depending on which is expected to be faster). This effectively compares the two different image elements centred on a point by finding a correlation coefficient for every possible pixel displacement within the base image element region. This can be thought of as a process of superposing the input image element, using its central correlation point pixel, over each pixel of the base image element and calculating the correlation coefficient based on how well the images match at each different overlay position. The resolution of this correlation process is limited by the maximum displacement of features between images. Thus, as the interval between images shortens, or the testing extension rate decreases, the possible resolution of the grid can increase, at the cost of requiring more images.

The elemental correlation coefficient distributions from the normalised 2D cross-correlation for each grid point are post-processed to eliminate any poor or undesirable correlation results. The peak correlation coefficient of each normalised elemental distribution corresponds to the displaced location of the current grid point from the base image to the input image. These displaced grid points make up the updated correlation point grid for the input image to be used as the base grid for the next incremental image pair. This process continues to loop through image pairs, updating changes to the correlation point grid with respect to the image time information until the whole image sequence has been analysed.

After the image correlations have been performed for every correlation point and for every pair of images in the sequence, the displacements of each correlation point corresponding to each image through time are known. Subsequently, the shear angle and effective strains in the fabric specimen are calculated from this known displacement field. The whole DIC code process is diagrammatically represented in Figure 1.

\section{Calculating shear strain}

In order to calculate the shear strain, adjacent points from the correlation point grid are first grouped to form either linear four-node or biquadratic nine-node elements depending on the desired degree of data smoothing. The nine-node elements are used in preference for this work since a greater number of grouped nodes are used in the calculation of elemental deformation. This reduces the effect of discontinuities or spurious nodal displacements. A set of nine quadratic shape functions can be used to determine a value at any point within the element based on contributions of values from all nine points that make up the element. Each element is defined using a $2 \mathrm{D}$ internal elemental coordinate system, $\xi(\xi, \eta)$, where $\xi$ and $\eta$ range from -1 to 1 across opposing walls of the element. Details of such elements and their shape functions are excluded from this work for brevity, but are commonly detailed in Finite Element theory texts [16]. Using the shape functions for these elements, the deformation gradient tensor, $\boldsymbol{F}$, is determined in terms of the elemental coordinate system $\xi(\xi, \eta)$ using the chain rule in Equation (1), where $\boldsymbol{x}(x, y)$ and $\boldsymbol{X}(X, Y)$ are the deformed and initial coordinates respectively. 


$$
\boldsymbol{F}=\frac{d \boldsymbol{x}}{d \boldsymbol{\xi}} \frac{d \boldsymbol{\xi}}{d \boldsymbol{X}}=\left[\begin{array}{ll}
\frac{d x}{d \xi} & \frac{d x}{d \eta} \\
\frac{d y}{d \xi} & \frac{d y}{d \eta}
\end{array}\right]\left[\begin{array}{ll}
\frac{d \xi}{d X} & \frac{d \xi}{d Y} \\
\frac{d \eta}{d X} & \frac{d \eta}{d Y}
\end{array}\right]=\left[\begin{array}{ll}
\frac{d x}{d \xi} & \frac{d x}{d \eta} \\
\frac{d y}{d \xi} & \frac{d y}{d \eta}
\end{array}\right]\left[\begin{array}{ll}
\frac{d X}{d \xi} & \frac{d X}{d \eta} \\
\frac{d Y}{d \xi} & \frac{d Y}{d \eta}
\end{array}\right]^{-1}
$$

From this the stretch tensor, $\boldsymbol{U}$, is calculated and the rotation tensor, $\boldsymbol{R}$, is determined from the polar decomposition shown in Equation (2).

$$
\boldsymbol{U}=\sqrt{\boldsymbol{F}^{T} \boldsymbol{F}}, \quad \boldsymbol{R}=\boldsymbol{F} \boldsymbol{U}^{-1}
$$

The following yarn tracking calculations are based on a hypoelastic fabric deformation model developed by Peng et al. [17] that has also seen recent success in drape modelling [3]. By considering a set of two vectors for the initial state of the yarn directions, $\boldsymbol{g}_{\boldsymbol{\alpha}}^{\mathbf{0}}$, a set of rotated vectors that remain orthogonal, $\boldsymbol{g}_{\boldsymbol{\alpha}}$, are then found by multiplication with the rotation tensor in Equation (3). Here $\alpha=1,2$ signifies the two independent principal yarn directions, and the zero superscript represents the initial undeformed state.

$$
\boldsymbol{g}_{\alpha}=\boldsymbol{R} \cdot \boldsymbol{g}_{\alpha}^{\mathbf{0}}
$$

Next the actual, deformed yarn directions, $\boldsymbol{f}_{\boldsymbol{\alpha}}$, are calculated from the initial yarn axes, $\boldsymbol{g}_{\boldsymbol{\alpha}}^{\mathbf{0}}$, and deformation gradient, $\boldsymbol{F}$, in Equation (4).

$$
\boldsymbol{f}_{\boldsymbol{\alpha}}=\frac{\boldsymbol{F} \cdot \boldsymbol{g}_{\alpha}^{\mathbf{0}}}{\left\|\boldsymbol{F} \cdot \boldsymbol{g}_{\alpha}^{\mathbf{0}}\right\|}
$$

The angles between the actual yarn axes, $\boldsymbol{f}_{\boldsymbol{\alpha}}$, and the simply rotated axes, $\boldsymbol{g}_{\boldsymbol{\alpha}}$, are calculated in Equations (5) and (6). An example of these vectors and angles is shown in Figure 2Error! Reference source not found. for the deformation of a single plain weave unit cell.

$$
\begin{aligned}
& \cos \theta_{\alpha}=\frac{\boldsymbol{g}_{\boldsymbol{\alpha}} \cdot \boldsymbol{f}_{\boldsymbol{\alpha}}}{\left\|\boldsymbol{g}_{\boldsymbol{\alpha}}\right\|\left\|\boldsymbol{f}_{\boldsymbol{\alpha}}\right\|} \\
& \sin \theta_{\alpha}=\frac{\boldsymbol{g}_{\boldsymbol{\alpha}} \times \boldsymbol{f}_{\boldsymbol{\alpha}}}{\left\|\boldsymbol{g}_{\boldsymbol{\alpha}}\right\|\left\|\boldsymbol{f}_{\boldsymbol{\alpha}}\right\|}
\end{aligned}
$$

Then finally the shear angle, $\gamma$, is calculated by finding the sum of the two angles in Equation (7), which are physically represented as the angles between each initial yarn axis and its corresponding deformed yarn direction.

$$
\gamma=\theta_{1}+\theta_{2}
$$

This is the shear angle resulting from the change between the initial state and the current state, at the centroid of each element in the formulation.

\section{Calculating effective yarn strains}

Since the true yarn directions are tracked throughout the image sequence, the DIC code is also capable of calculating the effective strains in each of the yarn directions. These effective strains can be used for further analysis of various test configurations but are not necessarily equivalent to the true yarn strains. Primarily, yarn slippage (where yarns slip relative to one another) can be a significant problem for the bias extension shear test that contributes to the effective yarn strains measured with DIC (since the DIC cannot differentiate between displacements caused by yarn slippage and yarn strain). This does however provide another measure for evaluating and comparing different test samples as is presented in this work. For the picture frame test however, yarn slippage is typically negligible and the effective yarn strains are expected to be representative of the true yarn strains. 
Normal strains from the stretch tensor, $\boldsymbol{U}$, can be converted from the global directions to the two yarn directions with the transformation matrix, $\boldsymbol{T}_{\boldsymbol{\alpha}}$, as described by Equation (9) and demonstrated in literature [3, 17]. Effective strains in each of the yarn directions are then $\varepsilon_{11}^{f_{1}}$ and $\varepsilon_{22}^{f_{2}}$.

$$
\begin{aligned}
\boldsymbol{T}_{\boldsymbol{\alpha}} & =\left[\begin{array}{cc}
\cos \theta_{\alpha} & -\sin \theta_{\alpha} \\
\sin \theta_{\alpha} & \cos \theta_{\alpha}
\end{array}\right], \quad \alpha=1,2 \\
\boldsymbol{U}^{\boldsymbol{f}_{\boldsymbol{\alpha}}} & =\left[\begin{array}{cc}
\varepsilon_{11}^{f_{\alpha}} & \varepsilon_{12}^{f_{\alpha}} \\
\varepsilon_{21}^{f_{\alpha}} & \varepsilon_{22}^{f_{\alpha}}
\end{array}\right]=\boldsymbol{T}_{\boldsymbol{\alpha}}{ }^{T} \boldsymbol{U} \boldsymbol{T}_{\boldsymbol{\alpha}}=\boldsymbol{T}_{\boldsymbol{\alpha}}{ }^{T}\left[\begin{array}{ll}
\varepsilon_{11} & \varepsilon_{12} \\
\varepsilon_{21} & \varepsilon_{22}
\end{array}\right] \boldsymbol{T}_{\boldsymbol{\alpha}}
\end{aligned}
$$

Ultimately, the advantage of the DIC code (programmed in MATLAB) which is made freely available by the authors [14], is that it can calculate and display shear angle distributions across samples specific to textile shear testing. Moreover, the design of the code facilitates the calculation of strains in the actual yarn directions, not simply in the assumed orthogonal material directions, which again is not an option in the more generalised DIC software packages. These values typically overestimate the true yarn strains, since the observed DIC strains also include any slippage in the yarn direction, however, they remain a useful analytical tool for DIC quality and slippage evaluation.

\section{Evaluation of bias extension samples using the new DIC approach}

\section{Method}

Among the prominent fabric shear tests, the bias extension method offers relative simplicity and good repeatability. Picture frame testing on the other hand, often requires a more complex rig, and has greater challenges in specimen alignment and clamping [18]. In the past, the bias extension method has often been criticised for difficulties in measuring shear strain or shear angle compared to methods like the picture frame test. However the use of the proposed DIC method mitigates this concern. It is also suggested that the range of shear angles in bias extension testing is more limited, and that yarn slippage can become prevalent [19]. Slippage though, occurs primarily at higher shear angles and can be neglected, since it tends not to occur inside the primary shear zone of interest [5], where the higher shear stresses help lock yarns in place.

Bias extension testing was performed under displacement control on an Instron 5948 MicroTester machine to achieve a high resolution and accuracy at lower loads. A loading rate of $10 \mathrm{~mm} / \mathrm{min}$ was used for these tests, as is typical, due to the large shear deformation range that is possible with woven materials. An aerospace grade carbon fibre fabric was used for this study, with plain weave architecture, $3 \mathrm{~K}$ tows and an areal density of $0.193 \mathrm{~kg} / \mathrm{m}^{2}$.

Two main specimen types for the bias extension test have been used in previous studies [4, 11] but little work has been reported on their relative performance. The first sample type was a narrow strip with a length recommended to be twice its width [4]. The second was a wider test specimen, with the width of the fabric extending beyond the clamp jaws. The wide samples were previously proposed to overcome limitations associated with the narrow sample geometry [11]. Both sample types were tested in this work, and exhibited the same central, diamond-shaped region of theoretically pure shear deformation. For narrow and wide samples with the same gauge length and clamp width, these central shear zones were similarly sized.

Narrow samples were cut into $50 \times 150 \mathrm{~mm}$ strips with yarns oriented $\pm 45^{\circ}$ to the taller, loading direction. Wide samples on the other hand were cut to twice the width, with dimensions of $100 \times 150 \mathrm{~mm}$ at the same orientation. Clamps required an area $50 \times 25 \mathrm{~mm}$ at both ends of the samples, therefore both sample types 
had a $50 \times 100 \mathrm{~mm}$ gauge area between clamps, with the wide samples having $25 \mathrm{~mm}$ extending from either side of the gauge area. Adhesive tape was used to reinforce the clamping area of the fabric samples and help prevent yarn slippage, however recent literature recommends an isosceles trapezoid shape for the clamping jaws that could be used to further prevent yarn slippage [20].Testing was conducted with five of each specimen configuration shown in Figure 3.

Preparation of test samples required particular attention due to the very low loads needed to disturb these fabrics from their initial state. As can be seen in Figure 3, silver markings were dotted onto areas of interest to enhance the capabilities of the DIC code to distinguish and track deformation (which becomes particularly difficult at high shear angles).

In order to facilitate the DIC analysis, a 12.2 Megapixel, Complementary Metal-Oxide-Semiconductor (CMOS) sensor camera was set up on a mount facing the test region. Images were taken at regular two second intervals to capture $0.33 \mathrm{~mm}$ changes in bias extension under displacement control. Advances in standard CMOS sensor technology have enabled high performance imaging that can be comparable to that of Charge Coupled Device (CCD) sensors at a reduced cost. For these bias extension experiments, performed in a controlled lab setting, CMOS sensors were more than adequate to support the high resolution DIC analysis.

\section{Raw bias extension results}

The raw load-extension results from the Instron 5948 MicroTester shows both sample types to be highly repeatable. These results are shown for two different load scales in Figure 4 with the overall mean curves in black. For all tests there was no observed fabric slippage from under the clamps (not to be confused with yarn slippage) since the loads were relatively low. The narrow samples produce bell-shaped curves with peak loads consistently reaching around $12 \mathrm{~N}$. Wide samples, on the other hand, show a steep curve with peak loads above $140 \mathrm{~N}$. Figure 4 shows that the wide bias extension samples are capable of achieving peak loads ten times greater than the narrow samples despite the consistent gauge lengths, clamping areas and number of gauge yarns ( $18 \times 18$ yarns in the central shear zone for both sample types). Some increase in loading is not unexpected, given the additional frictional resistance supplied by the excess material outside the gauge region in the wide samples. A slightly higher loading in wide samples at lower extensions supports this. However, the tenfold increase in peak loading, and divergence of sample behaviour above $20 \mathrm{~mm}$ extension in Figure 4, is attributed primarily to yarn slippage in the narrow samples.

During testing, the narrow samples initially displayed idealised shearing behaviour, though after $15-20 \mathrm{~mm}$ extension the central shear zone began to approach its in-plane deformation limit. Subsequently, yarn slippage was seen, and grew until it became the dominant mechanism for facilitating extension past $30 \mathrm{~mm}$, where the sample was no longer capable of sustaining load. These observations are reflected by the curves shown in Figure 4. Wide samples demonstrated similarly distinct shear zones during testing, with the central shear zone eventually approaching a locked state. However, shear deformation then caused out-of-plane buckling that was observed in the form of minor wrinkling past $20 \mathrm{~mm}$ extension. By $40-45 \mathrm{~mm}$ extension the wrinkling diminished again. This, combined with the drop in loading and divergence of test results in Figure 4, identifies the late onset of significant yarn slippage relative to the narrow samples which remained planar throughout testing.

\section{DIC results}


Images from all the bias extension tests were processed with the DIC code to quantify the shear deformation present in each sample. In order to validate and confirm the DIC results, manual digital measurements of the shear angle in the central shear zone were also taken for the comparison shown in Figure 5. There was generally good agreement, however at high shear angles the manual measurements became prone to greater error as yarns were increasingly difficult to distinguish with the human eye. This error was prevalent in the narrow sample results, though the problem was also encountered with the wide samples above $50^{\circ}$. As a result, the DIC shear angle predictions appeared to be more accurate than human measurements. This is not unexpected as subtle differences in tones and colours are objectively compared through DIC.

In Figure 5, narrow samples show dramatic stiffening behaviour for shear angles above $40^{\circ}$, ultimately appearing to asymptote at around $55^{\circ}$. Wide samples also begin to stiffen above $40^{\circ}$, but display asymptotic behaviour at $70^{\circ}$. The greater loads of the wide samples are also reflected in Figure 5 , though ultimately the narrow and wide results are very similar up to shear angles of around $55^{\circ}$.

The calculated results for both samples types are compared to the idealised kinematic theory for the bias extension experiment (neglecting effects such as yarn slippage) in Figure 6. Here the narrow and wide samples again show similar behaviour up to around $50^{\circ}$, slowly deviating from the basic theoretical curve. Past this point though, the narrow samples rapidly diverge, corresponding with the large degree of yarn slippage observed during testing. Wide samples, on the other hand, continue on the same near-linear trend until around $70^{\circ}$ before a similar divergence.

Full shear angle distribution results from DIC are shown in Figure 7 at increments of $10 \mathrm{~mm}$ extension, compared with actual images from testing. The general deformation of the DIC grid shows good agreement with the experimental shape of each of the samples for the complete range of bias extension. In both the narrow and wide shear angle distributions, there are clear signs of the three distinct shear regions commonly associated with bias extension testing. The triangular regions adjacent to the clamps exhibit negligible shearing, with peak values in the central shear zone that are twice that of the adjacent intermediate regions.

The DIC results do however exhibit some shear angle anomalies that are attributed to poor tracking and yarn slippage at $30 \mathrm{~mm}$ extension of the narrow samples. Increased shearing at the interface of the different shear zones in narrow samples is also observed, most clearly at $10 \mathrm{~mm}$ and $20 \mathrm{~mm}$ extension. It is likely that the increase of calculated shearing in the zone interfaces is representative of yarn slippage, since these are the areas where yarn slippage is well documented to occur [19]. Yarn slippage is also evidenced in the actual photos, where the narrow samples are seen to fray at the edges due to yarns slipping out from underneath each other.

The wide sample results in Figure 7 show far fewer local correlation errors or discontinuities, even at high extension. Similarly, the effective yarn strains for wide samples in Figure 8 are more consistent than for the narrow samples. Furthermore, the effective strain concentrations in narrow samples are greater for areas associated with yarn slippage. Some out-of-plane buckling behaviour has also been captured in Figure 7, where dark vertical lines of artificially increased shear angles correspond with wrinkling of the sample at $30 \mathrm{~mm}$ extension in the central shear zone. This behaviour does introduce some error into the results (peaking at around a $10 \%$ increase) since the DIC method employs a single camera system. However the mean DIC results in the central shear zone remain quite accurate overall, with an increased error of only around $5 \%$ for shear angle results above $65^{\circ}$. Significant out-of-plane bending behaviour also occurs in triangular regions either side 
of the central shear zone of the wide samples causing spurious DIC results, though most of this error can be neglected as it occurs outside the gauge area and region of interest.

\section{Normalisation of results}

For application in a draping model or for comparison against different tests, normalisation of the raw experimental shear angle, load and extension data is required. Normalisation procedures for narrow samples are well documented $[9,20]$, however several adjustments and assumptions have been made in order to normalise the wide sample results. Figure 9 shows the well known classification of idealised shear zones in a narrow sample: where region A experiences no shearing, region $C$ exhibits shear angles of value $\gamma$ and region B display partial shearing, $\gamma / 2$. Supported by DIC observations (see Figure 7 ), the area of region B in wide samples is assumed to be 2.5 times greater than that of the narrow samples for the purposes of normalisation. This is simply accounted for in the normalised shear force calculations with the addition of factor $a$ in Equation (10). Otherwise, the normalisation method is performed in accordance with the approach outlined in an international benchmarking effort for woven fabric material behaviour [9]. Region D in Figure 9 is assumed to provide negligible shear resistance (similar to region A), based on the experimental observation that out-ofplane buckling is the primary deformation mode.

$$
F_{s h}(\gamma)=\frac{1}{(2 H-3 W) \cos \gamma}\left[\left(\frac{H}{W}-1\right) F\left(\cos \frac{\gamma}{2}-\sin \frac{\gamma}{2}\right)-a W F_{s h}\left(\frac{\gamma}{2}\right) \cos \frac{\gamma}{2}\right]
$$

For narrow samples, the factor $a$ is 1 and wide samples it is 2.5 . These values for $a$ are based on the size of the idealised region $B$ in the two samples, since wide samples have 2.5 times the partial shear contribution from region $B$ than narrow samples. This approach for normalisation is iterative as it depends on the partial shear contribution from region B within the function for the central shear zone $\mathrm{C}, F_{s h}(\gamma)$. A typical approximation to initialise the iterative procedure is presented in Equation (11), where $b$ is 0.5 for narrow samples. For wide samples however, iterative stability is only achieved when $b$ is 0.75 , due to the geometric differences between the two sample types.

$$
F_{s h}\left(\frac{\gamma}{2}\right) \approx b \times F_{s h}(\gamma)
$$

The results of this normalisation procedure are shown in Figure 10. The two different sample types are seen to result in very similar curves up to around $55^{\circ}$, at which point the significant yarn slippage in narrow samples causes the results to diverge. In order to fully validate this normalisation approach for wide bias extension experiments, a more thorough investigation should be performed.

\section{Comparison of wide and narrow sample configurations}

The comparison of these two bias extension test samples suggests that wide samples may provide better results for characterisation purposes than the more commonly used narrow samples. The raw narrow sample loads were an order of magnitude smaller than the wide sample loads, and extensive yarn slippage was also observed in narrow tests. Wide samples were consistently more stable and capable of greater shear angles. The DIC results supported these observations, with indications of yarn slippage at an earlier onset in narrow samples, particularly when looking at the shear angle and effective yarn strain distributions. In general, the DIC visualisations demonstrate that slippage occurs in regions where yarns experience a high effective yarn strain in one direction but minimal effective yarn strain in the transverse direction, coupled with some shear deformation. The increased capability of the wide sample demonstrates that the bias extension test is not purely limited to lower shear angles as has been previously suggested by research using only narrow samples $[19,21]$. 
Ultimately it is the frictional effects in the peripheral fabric architecture of wide specimens that restricts and prevents yarn slippage. It is expected that this causes the difference of results between the two samples. Without yarn slippage to facilitate the extension of wide test samples, higher loads and shear angles are achievable, and out-of-plane bending effects are subsequently observed.

Despite the differences between the two specimen configurations, the normalised shear force results are very similar up to around $55^{\circ}$ for the wide and narrow samples; hence both appear to be appropriate for material characterisation in support of typical forming applications. Although, highly double-curved deformation may lead to high shear angles for which the wide sample would then be the more appropriate configuration.

\section{Conclusion}

This paper details a novel MATLAB-based Digital Image Correlation (DIC) code developed specifically for the movement of shear deformation in textile materials which accounts for non-orthogonal reorientation of the yarns. This low-cost approach tracks yarn orientations through deformation and is able to accurately measure shear angles from sequential images taken with a basic digital camera, along with calculating effective strains in the yarn directions, and produce detailed visualisations of the results. Accuracy and reliability of the DIC code have been validated against manually-measured experimental results from bias extension testing of an aerospace grade, carbon fibre plain weave fabric. As a further demonstration, the code has been used to evaluate and compare a more common narrow sample type with a wide sample type for bias extension experiments.

From observation, the wide samples showed a significant reduction in yarn slippage, an increase in the achievable shear angles (by over 25\%) and greater peak loads (by an order of magnitude). Out-of-plane wrinkling was also observed in the wide samples but not narrow samples, suggesting that the narrow samples may not have reached the true locking angle of the fabric. It is suggested that these benefits in the wide samples result from the additional frictional resistance in the material outside the gauge area.

\section{Acknowledgements}

This research was supported under the Australian Research Council's 'Linkage Projects' funding scheme (LP100100508) in partnership with Boeing Research \& Technology Australia. The second author would also like to acknowledge the financial support of Bombardier and the Royal Academy of Engineering.

\section{References}

1. Poe, C., Dexter, H. and Raju, I. (1999) Review of the NASA textile composites research. Journal of Aircraft. 36, 876-884.

2. Harrison, P., Abdiwi, F., Guo, Z., et al. (2012) Characterising the shear-tension coupling and wrinkling behaviour of woven engineering fabrics. Composites: Part A. 43, 903-914.

3. Khan, M.A., Mabrouki, T., Vidal-Sallé, E. and Boisse, P. (2010) Numerical and experimental analyses of woven composite reinforcement forming using a hypoelastic behaviour. Application to the double dome benchmark. Journal of Materials Processing Technology. 210, 378-388.

4. Wang, J., Page, J.R. and Paton, R. (1998) Experimental investigation of the draping properties of reinforcement fabrics. Composites Science and Technology. 58, 229-237.

5. Zhu, B., Yu, T.X. and Tao, X.M. (2007) Large deformation and slippage mechanism of plain woven composite in bias extension. Composites: Part A. 38, 1821-1828. 
6. Boisse, P., Gasser, A. and Hivet, G. (2001) Analyses of fabric tensile behaviour: determination of the biaxial tension-strain surfaces and their use in forming simulations. Composites: Part A. 32, 1395-1414.

7. Kawabata, S. (1980) The standardization and analysis of hand evaluation. The Textile Machinery Society of Japan, Osaka, Japan.

8. Culpin, M.F. (1979) The shearing of fabric: a novel approach. Journal of the Textile Institute. 70, 81-88.

9. Cao, J., Akkerman, R., Boisse, P., et al. (2008) Characterization of mechanical behaviour of woven fabrics: Experimental methods and benchmark results. Composites: Part A. 39, 1037-1053.

10. Lomov, S.V., Boisse, P., Deluycker, E., et al. (2008) Full-field strain measurements in textile deformability studies. Composites: Part A. 39, 1232-1244.

11. Potluri, P., Perez Ciurezu, D.A. and Ramgulam, R.B. (2006) Measurement of meso-scale shear deformations for modelling textile composites. Composites: Part A. 37, 303-314.

12. Willems, A., Lomov, S.V., Verpoest, I. and Vandepitte, D. (2009) Drape-ability characterization of textile composite reinforcements using digital image correlation. Optics and Lasers in Engineering. 47, 343-351.

13. Eberl, C. (2006) Digital image correlation and tracking - File exchange - MATLAB central. The MathWorks, Inc. http://www.mathworks.com.au/matlabcentral/fileexchange/12413-digital-image-correlation-andtracking.

14. Pierce, R.S. (2012) Shear strain DIC (for bias extension tests) - File exchange - MATLAB central. The MathWorks, Inc. http://www.mathworks.com.au/matlabcentral/fileexchange/39544-shear-strain-dic-forbias-extension-tests.

15. Lewis, J.P. (1995) Fast normalized cross-correlation. Vision interface. 10, 120-123.

16. Bathe, K.J. (2006) Finite Element Procedures. Cambridge, MA: Klaus-Jürgen Bathe.17. Peng, X.Q. and Cao, J. (2005) A continuum mechanics-based non-orthogonal constitutive model for woven composite fabrics. Composites: Part A. 36, 859-874.

18. Hivet, G. and Duong, A.V. (2010) A contribution to the analysis of the intrinsic shear behaviour of fabrics. Journal of Composite Materials. 45, 695-716.

19. Harrison, P., Clifford, M.J. and Long, A.C. (2002) Shear characterisation of woven textile composites. Proc. 10th European Conference on Composite Materials, Brugge, Belgium.

20. Härtel, F. and Harrison, P. (2014) Evaluation of normalisation methods for uniaxial bias extension tests on engineering fabrics. Composites: Part A. 67, 61-69.

21. Harrison, P., Clifford, M.J. and Long, A.C. (2004) Shear characterisation of viscous woven textile composites: a comparison between picture frame and bias extension experiments. Composites Science and Technology. 64, 1453-1465. 


\section{Figure Captions}

Figure 1: Flow diagram for the Digital Image Correlation (DIC) code.

Figure 2: Initial and deformed states of a plain weave unit cell, showing yarn rotation relative to a single cross over point.

Figure 3: Clamped shear test specimens with central shear zone highlighted, (a) narrow and, (b) wide.

Figure 4: Raw load-extension results from shear testing for narrow and wide samples; (a) full load scale and, (b) $1 / 10$ load scale.

Figure 5: Mean DIC results for shear angles in narrow and wide samples compared with manual measurements in the central shear zone.

Figure 6: Comparison of narrow and wide sample behaviour against idealised theory.

Figure 7: Results from bias extension testing at $10 \mathrm{~mm}, 20 \mathrm{~mm}$ and $30 \mathrm{~mm}$ extension: (a) narrow sample, (b) narrow DIC, (c) wide sample, and (d) wide DIC.

Figure 8: Effective strain DIC results from bias extension testing at $10 \mathrm{~mm}, 20 \mathrm{~mm}$ and $30 \mathrm{~mm}$ extension: (a) narrow warp, (b) narrow weft, (c) wide warp, and (d) wide weft.

Figure 9: Idealised geometry of narrow and wide bias extension samples for normalisation calculations.

Figure 10: Normalised shear force comparison for bias extension with narrow and wide sample types. 